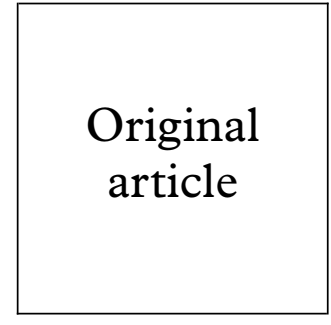

\title{
A randomised controlled trial comparing nonoxynol-9 lubricated condoms with silicone lubricated condoms for prophylaxis
}

\author{
R E Roddy, M Cordero, K A Ryan, J Figueroa
}

\begin{abstract}
Objective: We tested the effect of nonoxynol-9 (N-9) in condom lubrication on the risk of acquiring STD and genital discomfort.

Methods: The study was a triple masked, randomised controlled trial comparing N-9 lubricated condoms with plain silicone lubricated condoms among Dominican female sex workers.

Results: Randomisation provided two groups (313 for N-9 and 322 for plain) similar in baseline characteristics, but extensive loss to follow up occurred (56 women in each group completed the 24 week follow up). Most vaginal acts with clients were protected with condoms ( $99 \%$ of vaginal sex) but fewer acts with non-clients were protected (43\% of vaginal sex). No significant differences occurred in rates of cervical infections $(\mathrm{N}-9=3.4$ per 100 person months $v$ plain $=$ $2.8)$, trichomoniasis $(\mathrm{N}-9=2.8 v$ plain $=3.6)$, or discomfort rates $(\mathrm{N}-9=0.82 v$ plain $=0.92)$. Conclusions: Plain silicone lubricated condoms are as effective as $\mathrm{N}-9$ lubricated condoms, cost less, have longer expected shelf life, and therefore may be the better condom to provide. (Sex Transm Inf 1998;74:116-119)
\end{abstract}

Keywords: condoms; nonoxynol-9; silicone

Condoms are the foundation for programmes and individuals attempting to prevent the spread of sexually transmitted infections (STIs), including HIV. ${ }^{1}$ Several varieties of condoms are available and nonoxynol-9 (N-9) lubricated condoms have been promoted as providing extra protection because of the activity of N-9. However, N-9 lubricated condoms are more expensive than condoms lubricated only with silicone, must be packaged in foil instead of plastic, and have a shorter shelf life.

In general, in vivo studies have been supportive of the positive effects of condoms and N-9 when used separately. Observational studies of discordant couples show male condoms, used consistently, protect against STI. ${ }^{2}$ In addition, published randomised controlled trials indicate that $\mathrm{N}-9$ may reduce the risk of acquiring cervical chlamydial infection and gonorrhoea..$^{3-8}$

The evidence for a benefit of combining N-9 with the condom lubrication or as an addition to the inside of the condom is less compelling. One in vitro study simulated sexual intercourse and rupture of the condom. In this artificial setting, condoms coated in a $6.6 \% \mathrm{~N}-9$ lubricant preparation prevented growth, in appropriate media, of Chlamydia trachomatis, HSV type 2, and HIV. ${ }^{9}$ However, the tests conducted in the laboratory may not represent what happens with these $\mathrm{N}-9$ condoms during human coitus because rarely would the entire amount of N-9 inside a condom be available for use.

Public health agencies supplying condoms and information to prospective condom users for STI prophylaxis and contraception should have information about which type of condom to supply to get the most effective pregnancy and STI protection for the money spent. Our study was conducted to determine if N-9 lubricated condoms afford more protection from STI, and whether they produce more genital irritation than plain silicone lubricated condoms.

\section{Methods}

The study was a triple masked, randomised controlled trial. It was triple masked since the participants, the clinic staff, and the data analyst did not know which group was using the N-9 condoms. The study compared the cervical infection, trichomoniasis, and genital irritation rates of women individually assigned to the N-9 lubricated condom group with rates in women individually assigned to the silicone lubricated condom group. Using a rate difference of $20 \%$ for study size calculations, because N-9 lubricated condoms are about $20 \%$ more expensive, we had planned to recruit 700 women over a 1 year period. After 1 year of recruitment, we assessed the possibilities of continuing recruitment until the desired number of 700 was reached (635 had been recruited) against the probabilities of the rate difference achieving the $20 \%$ level, and decided to stop recruitment at that time. We attempted to follow each woman for 24 weeks or until she developed gonorrhoeal or chlamydial cervicitis. Women were scheduled to return for follow up visits every 2 weeks.

The randomisation list was generated by computer at Family Health International for each clinic. Random blocks of eight and 12 were used to ensure balanced assignment throughout the study. Sealed opaque envelopes were used to conceal the group assignment until the participant had been enrolled into the study. The assignment envelope was not opened by the clinic staff until the woman was 
Table 1 Frequency of characteristics found during enrolment examination by experimental group, and the prevalence ratio

\begin{tabular}{|c|c|c|c|}
\hline Characteristic & $\begin{array}{l}N-9 \text { condom } \\
\text { number }(\%) \\
n=313\end{array}$ & $\begin{array}{l}\text { Plain condom } \\
\text { number (\%) } \\
n=322\end{array}$ & $\begin{array}{l}\text { Prevalence ratio } \\
(95 \% \text { CI })\end{array}$ \\
\hline Abdominal pain with palpation & $27(9)$ & $27(8)$ & $1.0(0.6,1.7)$ \\
\hline \multicolumn{4}{|l|}{ Vulva: } \\
\hline erythema present & $4(1.3)$ & $5(1.6)$ & $0.8(0.2,3.0)$ \\
\hline ulcers or fissures present & $2(0.6)$ & $4(1.2)$ & $0.5(0.1,2.8)$ \\
\hline Vaginal discharge & $102(32.6)$ & $103(32.0)$ & $1.0(0.8,1.3)$ \\
\hline Vaginal inflammation & $2(0.3)$ & $5(1.6)$ & $0.4(0.1,2.1)$ \\
\hline Cervical ulcers present & $7(2.2)$ & $7(2.2)$ & $1.0(0.4,2.9)$ \\
\hline Trichomoniasis & $9(2.9)$ & $11(3.4)$ & $0.8(0.4,2.0)$ \\
\hline Candidiasis & $2(0.6)$ & $1(0.3)$ & $2.1(0.2,22.6)$ \\
\hline Gonorrhoea & $1(0.3)$ & $1(0.3)$ & $1.0(0.1,16.4)$ \\
\hline Chlamydia infection & $18(5.8)$ & $18(5.6)$ & $1.0(0.6,1.9)$ \\
\hline
\end{tabular}

These women did not contribute study time until after treatment and resolution of the problem.

found to be qualified for the study and had completed screening. No interim analysis was conducted and the field staff were unaware of any trends in the data. The envelopes were opened in consecutive order and the assignment slip was included in the records folder for each participant along with her signed consent form.

The study population was sex workers with multiple partners attending three STD clinics in Santo Domingo, Dominican Republic, aged $18-45$, free from signs or symptoms of sexually transmitted infections, or pregnancy (including 42 days post partum), with a normal pelvic examination, including bimanual examination. The women had to agree to use the study condoms exclusively for every act of vaginal intercourse possible, be willing to be interviewed about sexual activity, keep a pictorial coital log, and sign a consent form. The participants should not have taken an antibiotic in the week before enrolment. Because these women have multiple partners and frequent sexual intercourse, they represent a population of women with the highest exposure to STIs, potentially the most frequent use of $\mathrm{N}-9$ lubricated condoms, and the most to gain from the results of this study.

All condoms were made of latex, $52 \mathrm{~mm}$ in width, manufactured within 1 year of the start of the study, and were from the same lot. The N-9 lubricated condom had silicone lubrication with the addition of $32 \mathrm{mg}$ of N-9 at the time of packaging. The condoms used for comparison were silicone lubricated only. The condoms appeared identical and were packaged identically except for an A or B on the wrapper, so that neither the women nor the outcome assessors were aware of group assignment. Data entry was done without awareness of intervention group assignment. The data analyst was unaware of group assignment and analysed the data as group A and B; the masking was broken after the tables and statistics were completed (triple masked design).

Each woman was taught to apply a condom correctly using a penis model, and had to demonstrate the correct procedure before leaving the clinic. The participants were given as many condoms as they thought were needed for a 2 week period. At the initial visit, the number of condoms needed was ascertained by the woman estimating the number of sexual contacts she had had in the past 2 weeks and doubling the estimate. At follow up visits, the number of condoms needed was estimated from the coital logs and the number of condoms used in the previous 2 weeks. The goal of the condom distribution was to ensure that each woman had more than enough devices to use.

The screening, baseline, and follow up examinations consisted of vulvar, vaginal and cervical inspections, and bimanual examination. A vaginal specimen was collected for wet mount, an endocervical Gram stain was taken, and two endocervical specimens were collected-one for gonorrhoea culture, using a modified Thayer-Martin culture media, and one for a chlamydia test using Chlamydiazyme Diagnostic Kit (Abbott Laboratories). Because results from the gonorrhoea and chlamydia tests were not available on the day of enrolment, some of the women enrolled were infected. These women were continued in the study, treated, but did not contribute follow up time until a negative gonorrhoea culture or chlamydia test had been achieved. Women with genital ulcers at screening were not enrolled in the study until they had no signs of epithelial disruption.

Analysis used the intention to treat rule. Each participant was included in the group to which she was randomised, regardless of her compliance during follow up. Analysis began with a comparison of admission characteristics to assess the balance of randomisation. Univariable analysis examined the distributions of each variable, including baseline characteristics (age, marital status, birthplace, number of children, contraceptive usage, workplace, number of sexual partners, STI history, douching, and sexual behaviour). Prevalence ratios and 95\% confidence intervals assist with the comparison of the two experimental groups and were calculated using EPI INFO software. Rate ratios are the measures of effectiveness for the primary outcomes (cervical infection and trichomoniasis) and were calculated by dividing the incidence rate of the N-9 condom group by that of the silicone condom group using EPI INFO software. Data management and most analysis was conducted with SAS software (SAS Institute, Inc, Cary, NC, USA).

\section{Results}

We recruited and randomised 635 female sex workers between March 1994 and April 1995. The randomisation process yielded two comparable groups. The two groups were essentially the same for age, marital status, level of education, number of living children, number of sex partners in the 2 weeks preceding enrolment, number of vaginal sex acts in the 2 weeks preceding enrolment, whether or not chemicals or substances are inserted into the vagina, whether or not antibiotics were being used, and current method of contraception. The physical examination showed that the groups were similar for palpated abdominal pain, erythema or lesions on the vulva, vaginal discharge, vaginal inflammation, cervical lesions, trichomoniasis, candidiasis, gonorrhoea, and chlamydia infection (table 1). 
Table 2 Rates, rate ratios and 95\% confidence intervals (CI) for conditions that can be prevented by condom use or possibly increased by nonoxynol-9 use. The incidence rate is calculated using time to first infection or report of problem

\begin{tabular}{|c|c|c|c|}
\hline Condition & $\begin{array}{l}N-9 \text { condom } \\
n=182 \text { rate } \\
\text { (events } 100 \\
\text { woman months) }\end{array}$ & $\begin{array}{l}\text { Plain condom } \\
n=178 \text { rate } \\
\text { (events } 100 \\
\text { woman months) }\end{array}$ & Rate ratio $95 \% \mathrm{CI}$ \\
\hline Cervical or trichomoniasis infection & $6.22(44 / 7.07)$ & $6.66(46 / 6.91)$ & $0.93(0.63,1.41)$ \\
\hline Cervical infection & $3.7(25 / 7.42)$ & $2.76(21 / 7.62)$ & $1.22(0.68,2.18)$ \\
\hline Trichomoniasis & $2.82(20 / 7.10)$ & $3.62(25 / 6.91)$ & $0.78(0.43,1.10)$ \\
\hline Genital discomfort, reported & $0.82(6 / 7.34)$ & $0.92(7 / 7.58)$ & $0.89(0.30,2.63)$ \\
\hline Genital ulcers & $1.37(10 / 7.32)$ & $1.07(8 / 7.47)$ & $1.28(0.50,3.23)$ \\
\hline
\end{tabular}

Rate is per 100 woman months.

Cervical infection includes gonorrhoea and/or chlamydia infection.

Loss to follow up was extensive but evenly divided between the experimental groups. Of the 635 women randomised, 275 (43\%), did not contribute any follow up time, as they did not return to the clinic after enrolment. However, loss to follow up was similar for both $-42 \%$ for the N-9 group and $45 \%$ of the plain condom group. No participant was withdrawn because of an adverse experience. About $39 \%$ of the women contributed at least 3 months of follow up time- $41 \%$ for $\mathrm{N}-9$ and $37 \%$ for plain. One hundred and six participants remained in the study for the full 24 weeks, about $17 \%$ for both experimental groups.

We compared the three groups of women: (1) those who completed the study; (2) those who contributed follow up time; and (3) those who contributed no follow up time. Those who completed the study were older, had more children, had a lower number of sex partners at enrolment, reported fewer vaginal sexual contacts for the 2 weeks before study entry, and charged slightly less for sex.

Condom use was prevalent at baseline (the result of the screening criteria) and remained frequent and consistent throughout the study. Condom use was also comparable between the two groups during the study, $97 \%$ of coital acts in both groups for clients- $41 \%$ in the N-9 group and $45 \%$ in the plain condom group for regular partners.

None of the outcome measures demonstrated a clear clinical or statistical difference (table 2). When we put the STI outcomes (gonorrhoea, chlamydia infection, and trichomoniasis) together the rate ratio (RR) is 0.93 (95\% confidence interval (CI) $0.63-1.41$ ) indicating no effect. Looking separately at cervical infections $(\mathrm{RR}=1.22,95 \% \quad \mathrm{CI}=0.68$ 2.18) and vaginal infections, N-9 condoms may have been slightly better for vaginal infection, but not by much $(\mathrm{RR}=0.78,95 \%$ $\mathrm{CI}=0.43-1.1)$. We also did not find a clear indication that the N-9 was producing greater genital discomfort or problems. The rate of self reported discomfort attributed to the condoms did not differ $(\mathrm{RR}=0.89,95 \% \mathrm{CI}=0.3-2.63)$ and the physical examinations did not reveal a significant increase in genital lesions $(R R=$ $1.28,95 \% \mathrm{CI}=0.5-3.23$ ).

\section{Discussion}

The current study does not provide evidence for additional protection from cervical infection or trichomoniasis provided by condoms lubricated with N-9. Latex by itself is an effective barrier to infection. The situations when the N-9 may play a role in STI prevention would be in cases of condom breakage, leakage, or slippage. At these times, the amount of N-9 available at the vulnerable site of infection is unknown. Our findings do confirm that consistent condom use will produce low incidences of infection. The participants in this study had multiple partners and yet had a low incidence of gonorrhoea and chlamydial infection.

We were encouraged that no apparent increase in complaints of discomfort or signs of genital irritation were associated with $\mathrm{N}-9$ lubricated condoms. Our finding is consistent with data from another study of N-9 lubricated condoms. ${ }^{10}$ That investigation compared condoms with $0 \mathrm{mg}, 8 \mathrm{mg}$, and $16 \mathrm{mg}$ of N-9 for reported genital discomfort and effect on vaginal Gram stains. It found no differences in reported genital discomfort, but an increase in vaginal polymorphonuclear cells on Gram stain occurred in women using N-9 condoms.

The women who participated in our study are not representative of all female sex workers or women in general. We purposely screened our participants to select women who were going to be able to use condoms consistently because the purpose of the study was not to assess the effect of condom use versus no condom use, but consistent condom use of one type of condom versus consistent condom use of another type of condom.

A major limitation to interpretation of the data from the current trial is the extensive loss to follow up. For this reason, we consider this study to have the same evidential weight as a cohort observational study rather than a randomised controlled trial. ${ }^{11}$ While we demonstrate that the two groups are comparable (even after loss to follow up is taken into account), we cannot be certain of the effect that the loss to follow up has on the true measure of effect. We did achieve our goal of studying a group of sex workers who were consistent condom users. We suspect that the inconsistent users were lost to follow up. However, their reasons for not returning to the clinic may have been a condom failure that led to symptoms, causing the participant to seek health care at another clinic. Alternatively, the participant may have experienced an adverse effect and decided not to return to the clinic for further study. Any of these reasons may have biased the study findings in unknown directions depending on the frequencies in each group.

A question we do not address, but bears on the amount of protection provided by the N-9, is how much of the N-9 actually is available at the cervix to prevent infection? That is, how much of the N-9 is left in the package, left on the hands when the condom is donned, on the inside of the condom but not at the tip where the microbes are likely to be, or on the outside of the condom but rubbed off at the introitus as penetration occurs? These questions have important considerations concerning expectations of the additive effect of N-9 in condom lubrication, but may not be feasible to answer. 
In conclusion, the hypothesis that $\mathrm{N}-9$ added to condom lubricant will confer additional protection from cervical gonorrhoea, chlamydial infection, or both is not supported by these data. N-9 lubricated condoms cost more, have a shorter shelf life, and require special packaging when compared with plain lubricated condoms. Programmes buying large quantities of condoms may want to provide the less expensive type of condom until further evidence is available to support the use of N-9 lubricated condoms.

Informed consent was obtained from each participant, and the study and consent process were approved by the Protection of Human Subjects Committee at Family Health International for this study was provided by Family Health International with
funds from the United States Agency for International funds from the United States Agency for International reflect those of the funding agency.

We acknowledge the hard work of the study staff in the Dominican Republic and, in particular, Orquidea Ramirez, Maria Dignorah Ortiz, Marina Torres, and Ingrid Muñoz. We also thank Belinda Irsula of FHI for her assistance with monitoring the study.

1 Center for Disease Control and Prevention. Contraceptive method and condom use among women at risk for HIV infection and other sexually transmitted diseases. $M M W R$ 1996;45:820-3.
2 Feldblum PJ, Morrison CS, Roddy RE, Cates W Jr. The effectiveness of barrier methods of contraception in preventing the spread of HIV. AIDS 1995;9 (supp A):S85-S93

3 Cutler JC, Singh B, Carpenter U, et al. Vaginal contraceptives as prophylaxis against gonorrhea and other sexually transmitted diseases. Adv Plan Parent 1977;12:45-56.

4 Rosenberg MJ, Rojanapithayakorn W, Feldblum PJ, Higgin JE. Effect of the contraceptive sponge on chlamydial infection, gonorrhea, and candidiasis: a comparative clinical trial. $\mathscr{f} A M A$ 1987;257:2308-12.

5 Louv WC, Austin H, Alexander WJ, Stagno S, Cheeks J. A clinical trial of nonoxynol-9 for preventing gonococcal and clinical trial of nonoxynol-9 for preventing gonococcal

6 Niruthisard S, Roddy RE, Chutivongse S. Use of nonoxynol-9 and reduction in rate of gonococcal and nonoxynol-9 and reduction in rate of gonococcal and

7 Rendon AL, Covarrubias J, McCarney KE, et al. A controlRendon AL, Covarrubias J, McCarney KE, et al. A control-
led, comparative study of phenylmercuric acetate, nonoxynol-9 and placebo vaginal suppositories as prophylactic agents against gonorrhea. Curr Ther Res 1980;27 780-3.

8 Kreiss J, Hgugi E, Holmes KK, et al. Efficacy of nonoxynol-9 contraceptive sponge use in preventing heterosexual acquisition of HIV in Nairobi prostitutes. ҰAMA 1992;268:477-82.

9 Judson FN, Ehret JM, Bodin GF, Levin MJ, Rietmeijer CA In vitro evaluations of condoms with and without nonoxynol-9 as physical and chemical barriers against Chlamydia trachomatis, herpes simplex virus type 2, and human immunodeficiency virus. Sex Transm Dis 1989;16: 51-6.

10 Ward $H$, De Lacourt A, Kitchen B. Nonoxynol-9 in lubricated condoms: results of a study in female prostitutes. Sex Transm Dis 1996;23:413-4.

11 US Preventive Services Task Force. Guide to clinical preventive services. 2nd ed. Baltimore: Williams \& Wilkins, 1996 862 